\title{
RESIDUAL NILPOTENCE AND RELATIONS IN FREE GROUPS
}

\author{
BY GILBERT BAUMSLAG ${ }^{1}$ AND ARTHUR STEINBERG \\ Communicated by R. C. Lyndon, January 7, 1964
}

Introduction. Relations between elements of a free group lead directly to identities for all groups, which are of considerable importance. In 1958 R. C. Lyndon [7] initiated a study of relations in free groups; in particular Lyndon proved that if $g_{1}, g_{2}, g$ are elements of a free group and

$$
g_{1}^{2} g_{2}^{2}=g^{2}
$$

then $g_{1}, g_{2}, g$ generate a cyclic group. Since then a number of generalizations of this theorem have been obtained by E. Schenkman [10], John Stallings [12], and Gilbert Baumslag $[1 ; 2]$. The most recent result of this kind proved by M. P. Schützenberger [11] (cf. also R. C. Lyndon and M. P. Schützenberger [8]) and, independently, also by Arthur Steinberg [13], states that if

$$
g_{1}^{p} g_{2}^{q}=g^{r},
$$

where now $p, q$ and $r$ are integers greater than 1 , then again $g_{1}, g_{2}, g$ generate a cyclic group.

Similarly, if instead

$$
g_{1}^{-1} g_{2}^{-1} g_{1} g_{2}=g^{r} \quad(r>1),
$$

then once more $g_{1}, g_{2}, g$ generate a cyclic group (M. P. Schützenberger [11], Gilbert Baumslag [3], and A. Karass, W. Magnus and D. Solitar [6], and Arthur Steinberg [13]).

The purpose of this note is to announce the following theorem which contains both the aforementioned theorems as special cases.

THEOREM 1. Let $w=w\left(x_{1}, x_{2}, \cdots, x_{n}\right)$ be an element of a free group $F$ freely generated by $x_{1}, x_{2}, \cdots, x_{n}$ which is neither a proper power nor a primitive. ${ }^{2}$ If $g_{1}, g_{2}, \cdots, g_{n}, g$ are elements of a free group connected by the relation

$$
w\left(g_{1}, g_{2}, \cdots, g_{n}\right)=g^{m} \quad(m>1),
$$

then the rank of the group generated by $g_{1}, g_{2}, \cdots, g_{n}, g$ is at most $n-1$.

${ }^{2}$ This work has been supported by a grant from the National Science Foundation, Grant G. P. 27.

2 An element in a free group is termed primitive if it can be included in a set of free generators. 
The authors would like to justify this joint announcement of work done independently by pointing out that Theorem 1 was obtained first by Baumslag although a more general result was already implicit in the Ph.D. thesis of Steinberg.

It is worth noting that the two proofs of Theorem 1 are completely different. On the one hand, Steinberg makes use of the Freiheitssatz of Wilhelm Magnus [9]. The information obtained in this way is so precise that a more general result than Theorem 1 can be obtained. Baumslag's proof, on the other hand, is less incisive, making use of residual properties and groups with unique roots (cf. $[4 ; 5]$ ). However, it seems likely that this approach might well be of value in treating analogous questions for other varieties of groups. Consequently, both proofs are of independent interest; they will appear separately, in detail, elsewhere.

\section{REFERENCES}

1. Gilbert Baumslag, On a problem of Lyndon, J. London Math. Soc. 35 (1960), 30-32.

2. - On generalised free products, Math. Z. 78 (1962), 423-438. 303.

3. - Some aspects of groups with unique roots, Acta Math. 104 (1960), 217-

4. - Some remarks on nilpotent groups with roots, Proc. Amer. Math. Soc. 12 (1961), 262-267.

5. K. W. Gruenberg, Residual properties of infinite soluble groups, Proc. London Math. Soc. (3) 7 (1957), 29-62.

6. A. Karass, W. Magnus and D. Solitar, Elements of finite order in groups with a single defining relation, Comm. Pure Appl. Math. 13 (1960), 57-66.

7. R. C. Lyndon, The equation $a^{2} b^{2}=c^{2}$ in free groups, Michigan Math. J. 6 (1959), 89-95.

8. R. C. Lyndon and M. P. Schützenberger, The equation $a^{m}=b^{n} c^{p}$ in a free group, Michigan Math. J. 9 (1962), 289-298.

9. W. Magnus, Ueber diskontinuierliche Gruppen mit einer definierenden Relation (Der Freiheitssatz), J. Reine Angew. Math. 163 (1930), 141-165.

10. E. Schenkman, The equation $a^{n} b^{n}=c^{n}$ in a free group, Ann. of Math. (2) 70 (1959), 562-564.

11. M. P. Schützenberger, Sur l'équation $a^{2+n}=b^{2+m} c^{2+p}$ dans un groupe libre, C. R. Acad. Sci. Paris 248 (1959), 2435-2436.

12. John Stallings, On certain relations in free groups, Notices Amer. Math. Soc. 6 (1959), 532.

13. Arthur Steinberg, Ph.D. thesis, New York University, 1962.

NeW YORK UNIVERSITY AND Fairleigh Dickinson University 